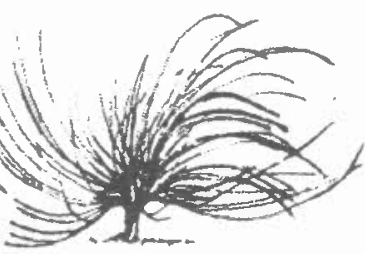

\title{
El libro de texto: los profesores de hoy
}

José Pablo Céspedes ${ }^{l}$ Universidad Nacional, Costa Rica

Heredia, Costa Rica jpcg1202@yahoo.com

Edwin Andrés Pérez ${ }^{3}$ Universidad Nacional, Costa Rica Heredia, Costa Rica andresbribri@gmail.com
Jean Carlo Vargas ${ }^{2}$

Universidad Nacional, Costa Rica

Heredia, Costa Rica ensayandoenlauna@gmail.com

Adrián Chavarría Universidad Nacional, Costa Rica Heredia, Costa Rica adrian_13_12chava@hotmail.com

\begin{abstract}
Resumen
Este artículo es una síntesis de una investigación realizada con el afán de comprender e interpretar la realidad de la función que cumplen los libros de texto dentro de los salones de clase, donde se evidenció lo innecesario de operar con éstos para llevar a cabo el proceso de enseñanza y de aprendizaje. Los resultados del estudio fueron obtenidos en un colegio privado de la provincia de Limón, el cual alberga una población de clase media. Este colegio posee una infraestructura en óptimas condiciones y a su vez los padres de familia cancelan una mensualidad elevada, pero la calidad de educación se asemeja a la de un colegio público, ya que los contenidos curriculares que se enseñan no corresponden a los objetivos que pretenden alcanzar dichos centros educativos.
\end{abstract}

Recibido: 08 de setiembre, 2010 - Aprobado: 29 de junio, 2011

Estudiante de la carrera de Estudios Sociales de la Universidad Nacional. Estudiante de la carrera de Estudios Sociales de la Universidad Nacional. Estudiante de la carrera de Estudios Sociales de la Universidad Nacional.

Estudiante de la carrera de Estudios Sociales de la Universidad Nacional. 
Palabras claves: Libros de texto, proceso de enseñanza y aprendizaje, recursos didácticos, metodología, modelo pedagógico.

\begin{abstract}
This is a research synthesis done with the goal of comprehending and interpreting the real role of textbooks in the classrooms. This paper shows the unnecessary usage of textbooks for conducting the teaching-learning process. The study results were obtained at a private high school in Limon province which holds a medium-class population. Nonetheless, this institution possesses an excellent infrastructure and parents pay a high fare, the educational quality resembles a public school one. This is due to the lack of correspondence between the curricular content and the teaching objectives that are established for this type of centers.
\end{abstract}

Keywords: Textbook, teaching and learning process, teaching materials, methodology, and pedagogical model.

\title{
Introducción
}

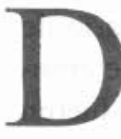

urante los últimos 50 años ha habido varias colecciones de libros de texto, los cuales han sido escritos por diversos equipos de autores y publicados por diferentes editoriales.

Entre las casas editoriales que se prefieren por la mayoría de los colegios tanto públicos como privados, se encuentra la editorial EDUVISION, lo que influye en su preferencia es que ofrece una serie de recursos didácticos, tanto para el beneficio del centro educativo, profesores como estudiantes. Entre los que podemos destacar están: películas o documentales en formato DVD, giras, capacitación para los profesores y otros recursos adicionales.

El Ministerio de Educación Pública (MEP) es el encargado de realizar toda esta elaboración de los programas de estudio que utilizan las casas editoriales para la construcción de los libros. Así mismo, dentro de este marco regulador existen los programas que guían, orientan y dan las disposiciones a instituciones, las cuales deben de cubrir los contenidos establecidos en éstos, con el fin de estandarizar la educación para que al final el resultado sea común. 
Estos programas son impartidos generalmente por los profesores que se manejan dentro de un paradigma y modelo pedagógico; el paradigma "es una sólida red de compromisos conceptuales, teóricos, instrumentales y metodológicos; incluye: un cuerpo explícito de creencias, teóricas y metodológicas entretejidas que permiten la selección, evaluación y crítica, es la fuente de los métodos, problemas y normas de solución aceptados por cualquier comunidad científica" (Echeverria, 2007).

Se selecciona un modelo pedagógico del cual se apropia el profesor de acuerdo a su formación universitaria y experiencias de vida. Esto se llega a poner en práctica tanto en el aula primaria como en secundaria, este es un lugar en donde se llega a impartir clases, transmitir información o construir conocimientos.

El profesor es el encargado de formar un ser integral tomando como eje los contenidos dados por el Ministerio de Educación Pública. La manera de impartir estos contenidos pueden afectar a los estudiantes en la adquisición de sus conocimientos, donde el aprendizaje puede llegar a ser significativo o no significativo, a la vez éstos van a ser el elemento fundamental dentro del sistema.

La relación entre profesor y estudiante se da mediante distintas herramientas tradicionales o no tradicionales, estas técnicas son indispensables para realizar el proceso de enseñanza y de aprendizaje. Estos serán los elementos que el profesor utilizará para su mediación pedagógica, la cual se define como "el tratamiento de contenidos y de las formas de expresión de los diferentes temas a fin de hacer posible el acto educativo, dentro del horizonte de una educación concebida como participación, creatividad, expresividad y relacionalidad" (Castillo, 1999).

El libro de texto, según EDUVISION, "Es un conjunto de temas referentes a historia y geografia, que constituye una valiosa guía a los estudiantes para que puedan prepararse adecuadamente en su formación integral" (EDUVISIÓN, 2005). Éste puede ser uno de estos instrumentos, en donde su utilidad va a depender del modelo pedagógico y la relación con éste va a dar su respectivo uso que puede ser positivo o negativo.

\section{Metodología}

La investigación en la que se basa éste articulo esta dentro del paradigma naturalista con enfoque cualitativo, dado que se pretende sustituir las nociones científicas de predicción y control por elementos 
de comprensión, significado y acción. También utiliza la recolección de datos sin medición numérica para descubrir o afinar preguntas de investigación en el proceso de interpretación. (Hernandez, 2006).

En ese proceso investigativo el sujeto y el objeto de estudio se relacionan para construir conocimiento, insertándose en el mundo de los sujetos participantes y no participantes, se interesa por el estudio de las diferencias y peculiaridades determinadas por el contexto, por lo que se acepta que la realidad es dinámica, múltiple, holística, inductiva y orientada al proceso.

Por ende, la finalidad de la investigación es comprender e interpretar la relación sujeto-objeto, que es de completa dependencia, ya que se afectan mutuamente. Ambos se modifican conforme se van pasando las etapas de investigación, lo que beneficia a una retroalimentación constante, aquí no se toma como relevante la relación causa-efecto, sino que se busca descubrir o generar teorías.

El trabajo de campo consiste en una participación intensa, en largos periodos de tiempo con los sujetos en estudio, por lo que se requiere de un registro detallado de todos los acontecimientos, al mismo tiempo su análisis es minucioso, para analizar esto se usa como criterio de calidad la triangulación.

La recolección de datos puede realizarse de diferentes formas, se utiliza: la entrevista en profundidad, la observación no participante y el cuestionario para llegar a comprender y desarrollar los objetivos. Éstos no son rígidos ni preestablecidos, sino abiertos, emergentes y nunca completos y cerrados.

Estos instrumentos son técnicas cualitativas, en la que se usa la grabadora como medio de recopilación de información acompañado de opiniones y comentarios que puedan mostrar datos relevantes para el desarrollo de la investigación. Se analizan mediante técnicas como: la triangulación, reflexión personal, el análisis crítico, la contrastación de hipótesis, por consiguiente el presente artículo va a ser descriptivo e inductivo y se dará énfasis en los hechos suscitados en el salón de clases.

\section{Descripción de la Institución}

La Institución en la que se realiza la investigación fue fundada en 1950, para abrirle una puerta de enseñanza-aprendizaje a la comunidad 
limonense, enfatizando la religión católica, posee buena infraestructura para albergar más de 500 estudiantes.

La institución comprende los siguientes niveles de enseñanza: educación preescolar, primer y segundo ciclo (Primaria), tercer ciclo y educación diversificada de la educación general básica (Secundaria), posee un total de 524 estudiantes matriculados, de los cuales cuarenta y cuatro son becados y poseen ayuda socioeconómica de $20 \%-25 \%$ sobre la mensualidad.

Concerniente a la infraestructura física, cuenta con 21 aulas disponibles, los espacios complementarios que dispone el centro son: las oficinas administrativas, gimnasio multiuso, tarima para actos cívicos, soda, librería, biblioteca, cuenta con tres laboratorios uno para computación, inglés y química.

Abrieron un aula audiovisual, esta tiene una pizarra interactiva, pero el colegio ha adquirido tres pizarras interactivas más, las cuales se encuentran en el área de preescolar, en el laboratorio de inglés y de cómputo, esto con el fin de facilitar los procesos de enseñanza y de aprendizaje de los estudiantes.

En lo que se refiere a áreas recreativas cuenta con: comedor, cancha de fútbol, zona verde en la cual se encuentran varios plays, áreas para que los estudiantes coman, hagan actividades y que estén en contacto permanente con la naturaleza.

La institución aplica los planes de estudio del Ministerio de Educación Pública en aquellas materias correspondientes a las del currículo del MEP y planes propios en aquellas materias del currículo institucional que se imparten más allá del plan de estudio. Posee reglamento interno tanto para el personal y los estudiantes y aplica el instructivo de evaluación dispuesto por la propia normativa interna, no acatan la del Ministerio de Educación.

\section{Descripción de la investigación}

Para conformar la investigación, el grupo se dirigió a la provincia de Limón en varias ocasiones para realizar la entrevista y la observación al grupo respectivo. Una vez en el colegio se empezó por hablar con los encargados de éste y explicarles el motivo de nuestra visita e investigación. 
Ya con el permiso de la Hermana Directora, el grupo se dispuso a buscar a algún (a) profesor (a) que estuviera anuente a ayudarnos con nuestra investigación, esta fue la profesora de la sección 10-2, la cual después de explicarle el motivo de nuestra visita nos tendió la mano con mucho gusto.

Se empezó la investigación primero con observaciones, esto para conocer al grupo y que ellos no nos vieran como gentes extraños a la hora de ahondar en la investigación. Para esos momentos utilizamos un instrumento de observación no participante, elaborado por el grupo, en donde se recogió información referente a la utilidad que le daba el profesor y los estudiantes al libro de texto, los recursos didácticos, la evacuación de dudas por parte del profesor, la criticidad y análisis de las ideas del libro de texto por parte de los estudiantes, entre otros.

Después de las observaciones nos enfocamos en hacerle una entrevista estructurada a la profesora con temas relacionados al modelo pedagógico que utilizaba en sus lecciones, las diferentes teorías del aprendizaje, la utilización de recursos y técnicas didácticas, el papel del libro de texto en el salón de clase y la relación docente-estudiante. Todo esto nos serviría para conocer más a fondo el tipo de profesor con el que estábamos trabajando y el papel que jugaba el libro de texto que es el principal protagonista de esta investigación.

Con las observaciones previas y la entrevista estructurada al profesor, sólo nos faltaba la opinión de los estudiantes para poder darle cuerpo al trabajo. Para ello utilizamos un cuestionario de preguntas abiertas, en donde se conoció la opinión de los colegiales respecto a la utilización del libro de texto por parte del profesor, algunas sugerencias para sacarle mayor provecho, cómo se sienten con la relación estudiante-docente y el papel del educador ante el libro de texto, entre otras.

Con esto tres componentes, el grupo se dispuso a formar su investigación para la cual conformó un cuadro de recopilación de la información de cada uno de los instrumentos utilizados (matrices), éste fue muy útil para obtener las conclusiones y elementos más importantes para la indagación.

\section{Descripción grupo de investigación}

El grupo de investigación fue un decimo y se compone de un total de 21 estudiantes, de los cuales, 12 son mujeres y 9 hombres. 
Los estudiantes se consideraron muy responsables, disciplinados y participantes, solo uno de ellos se perfiló como irresponsable. Respecto a la religión, un $90 \%$ de los estudiantes profesan la Religión Católica y un 10\% la Católica Anglicana.

La mayoria de los estudiantes viven en el Cantón Central de la Provincia de Limón, en los siguientes barrios: Siglo XXI, Corales 2 y 3, Pueblo Nuevo, Rossvelth, La Colina, Cieneguita, Limoncito, Moín, Barrio Veracruz, Pacuare, Cocos, Lomas de Chita, solo dos de los estudiantes viven fuera del casco central, ellos residen en las comunidades de Rio Banano y Liverpool.

Respecto al estrato social todos los alumnos, respondieron que pertenecen a la Clase Media limonense; los alumnos en un $90 \%$ no trabajan, en cambio el $10 \%$ trabajan en negocios familiares.

\section{La utilización del libro de texto}

El libro de texto se presenta como un sustituto del docente, en donde se convierte en un ente hegemónico poseedor de toda la verdad. Es idolatrado por el docente y por alumnado. Lo que abría que preguntarse es ¿Cuáles son los factores que permiten que el libro sobrepase las facultades del docente?, esto se puede analizar desde dos perspectivas la del docente y la del alumno, pues no son las mismas, hay diferentes usos y finalidades.

\section{Papel del docente en la utilización del libro de texto.}

El docente utiliza el libro como su único medio didáctico de enseñanza, e incluso se convierte en su marco de referencia, el papel del docente se reduce a ser un simple trasmisor de lo que dice y se plasma en éste libro, el positivismo está muy arraigado sin siquiera saberlo, ni conocerlo, pareciera que estas prácticas son usuales y frecuentes tanto en colegios públicos como privados, diurnos y nocturnos; esta es una deducción tanto de la investigación como de anteriores trabajos efectuados en otros cursos pertenecientes a la carrera.

Corresponde a la formación del docente y en la concepción ética que practique, el uso que le dé al libro de texto, no debe ser la intención de un docente perjudicar o hacer inútil el conocimiento si no que es una cuestión implantada dentro de su mentalidad de la misma forma que 
instaura a los estudiantes el conocimiento, por lo cual esto no es más que expresión de ignorancia y vagancia.

La ignorancia no deja ver ni las causas ni las consecuencias de su actuar, por ende no hay análisis, ni introspección de la función y utilidad de su labor, es algo rutinario y sin trascendencia, es algo mecánico.

La vagancia cobra vida cuando no hay interés del docente de asesorarse más allá, incluso algunas editoriales ofrecen la actualización no participa, más bien es algo casi satanizado, más allá que de lo que se enseña en una universidad no hay nada más, las lecturas y los conocimientos del docente parecieran expresarse en función de un título y no del conocimiento lo cual se evidencia en la falta de lectura.

El libro predomina en el aula a pesar de intentos de la docente de trabajar sin este, siempre toda actividad o dinámica que se llega a realizar esta relacionada de forma directa o indirecta con el libro, por lo que se concluye que el libro tiene la tutela de la clase y en base a este gira y se desarrolla la clase. Fue convertido por la profesora en el ente hegemónico y poseedor de toda la verdad y a su vez los alumnos absorbieron sumisamente esta idea o imposición.

La reacción de los estudiantes ante la utilización del libro de texto en su mayoría todos son sumisos, dóciles, pero esto no quiere decir que todos no están subyugados a estas actividades de mediación, sino que hay educandos que se manifiestan de diversas maneras entre las cuales están: la reacción con una mala actitud, cansado de hacer siempre lo mismo, no hay motivación ni interés, es una cuestión memorística y tienen completamente adoptada la rutina.

No obstante, la idolatración del libro tanto por parte de los estudiantes como de la profesora, denota que dentro de todo este sistema mecanizado y de estrecha relación con el libro de texto, hay una inconformidad en algunos estudiantes, que nos indican, que el libro no llega a saciar su demanda de conocimiento y aprendizaje, exigen otro tipo de material, quieren una alternativa más dinámica y más significativa.

A raíz de todo lo anteriormente dicho por los estudiantes, éstos optan por cambiar la forma para poder sacarle un mayor provecho al libro de texto. Sin embargo, las propuestas planteadas por los educandos siempre se inclinan directa o indirectamente al uso del libro, debido a que proponen...

La profesora recomienda el libro al final de año a pedido de la dirección, éste es impuesto por el profesor que estuvo el año anterior 
denotándose una tendencia a usar el libro de texto desde los máximos encargados.

Incluso ya está estandarizado la estructura que debe tener el libro en relación con el año, por ejemplo, para Quinto Año la profesora indica que éste debe contener prácticas y esquemas, ya que esto es lo que más les interesa a ellos para prepararlos para que en los resultados de las pruebas de Bachillerato sean del cien por ciento, pues además es con esto que miden su desempeño laboral.

Referente a la mediación pedagógica utilizada por la profesora dentro del salón de clases, según en la observación grupal no participativa, se constató que ella recurre a la pizarra, para colocar el tema a tratar y pone las palabras clave para guiar el trabajo del cuestionario, para responder éste los estudiantes se basan en el libro de texto.

Si nos referimos a la evacuación de dudas el estudiante se muestra con una postura contradictoria al no preguntar durante la clase, pues prefieren y se concentran en trascribir lo que está en el libro, las dudas surgen con la aproximación del examen.

En el caso del docente cuando tiene que abarcar dudas no se despega del libro, ya que a pesar de que evacua las mismas con su conocimiento éste no es más que una réplica del libro que no trasciende a más de lo que él está plasmado, igualmente para brindar un ejemplo se hace con base en el libro. La percepción del docente por parte del estudiante es que éste utiliza los dos métodos tanto el libro como su propio conocimiento para evacuar las dudas.

En relación con las actividades de mediación del docente de Estudios Sociales vistas en la observación utiliza el cuestionario como su principal herramienta didáctica para dar la clase. La profesora no manejaba el concepto de modelo pedagógico, ni siquiera lo conocía, pero se contradijo cuando decía que "utilizaba una combinación de ambos, que el modelo empleado era en relación al nivel que se impartía, indicaba: "con décimo soy positivista, soy dictadora", la conclusión es que "se trabaja según el nivel, porque hay que sacar la tarea no se puede ser constructivista porque el tiempo no da".

Debido a que es una técnica que la han utilizado durante toda su vida durante su formación, esta actividad la realizan para ganarse los puntos del trabajo cotidiano y una respuesta más evidente se refleja en la conducta del estudiante, cuando éste se muestra disconforme, aburrido, con pereza, sin motivación y no está anuente a recibir las clases. 
La relación entre el docente-estudiante se basa en el respeto y no en el miedo, según se observó, pues los estudiantes pueden participar sin temor. La profesora maneja las situaciones extra curriculares de los estudiantes, conoce el contexto en el que viven y se relacionan fuera de la institución, sin embargo no sabe cómo brindar la ayuda que éste requiere.

Por otro lado, existe una disciplina diferenciada a partir de los resultados, cada estudiante esta etiquetado por su eficacia o destreza para finalizar los trabajos en la hora indicada, con base en esto se aplica una disciplina libre o estricta. La mayoría de los estudiantes conciben la relación como una cuestión vertical y jerarquizada, donde la profesora esta por arriba de ellos y la comunicación es buena.

Lo que nos pueden arrojar e indicar este trabajo es que el libro de texto predomina en el salón de clase en esta institución, bajo el modelo positivista, pues muchos profesores lo ven como un medio que les facilita el trabajo y no como lo que es un simple material que hay que saber utilizar pues es inerte por sí solo.

Las casas editoriales que se escogen en las instituciones prevalecen no por su calidad si no por ciertos beneficios que dan como las capacitaciones, aunque estas capacitaciones se reducen a indicar la mejor manera de utilizar los productos que ellos mismos ofrecen, pero en realidad lo que proponen es una alternativa donde el papel del docente se ve reducido a un simple trasmisor y explicador de lo que dice el libro.

\section{Conclusiones}

Este artículo permite comprender e interpretar la realidad en cuanto al modelo pedagógico en relación a los materiales didácticos empleados y de la forma en que los perciben tanto el docente como el alumnado.

La realidad es que el libro de texto no tiene ningún problema, no hay necesidad de satanizarlo, porque no es él, el que está mal, sino la utilización que le da el docente que tiene estrecha relación con su modelo pedagógico, porque una cosa es que el libro tenga la última palabra y otra que sea un recurso complementario en el proceso de enseñanza y de aprendizaje. 


\section{REFERENCIAS}

Castillo, F. G. (1999). La Mediación Pedagógica. Apuntes para una educación a distancia alternativa. Buenos Aires, Argentina: CICCUS-La Crujía.

Echeverria, R. B. (2007). Investigacion un camino al conocimiento. San José: EUNED. EDUVISIÓN. (2005). Nuevos Horizontes 10 y 11 . San José, Costa Rica: EDUVISIÓN.

García, C. S. (2002). Otros Amenazantes. San José: Universidad de Costa Rica.

Gómez, G. R. (1996). Metodología de la Investigación Cualitativa. España: Aljibe.

González, O. (1996). El enfoque histórico cultural como fundamentación de una concepción pedagógica. Ibagué, Colombia: El Poiras.

Hernández, R. (2006). Metodologia de la Investigacion. Mexico DF: Mc Graw-Hill Interamericana.

http://www.icer.co.cr/ICER/icer.html. (2010). Instituto Costarricense de Enseñanza Radiofónica. San José: ICER.

http://www.mep.go.cr/. (2010). Misión Institucional. San José.

http://www.santillana.co.cr/. (2010). Grupo Santillana. San José: Santillana.

Reyes, A. (1999). Técnicas y modelos de calidad en el salón de clases. México D.F.: Trillas.

Tristá, B. (1985). Dirección en los centros de educación superior. La Habana: MES 